\title{
Ultrahigh finesse Fabry-Pérot superconducting resonator
}

\author{
S. Kuhr, ${ }^{\text {a) }}$ S. Gleyzes, C. Guerlin, J. Bernu, U. B. Hoff, S. Deléglise, S. Osnaghi, \\ M. Brune, and J.-M. Raimond ${ }^{b)}$ \\ Laboratoire Kastler Brossel, Ecole Normale Supérieure, CNRS, University Paris 6, 24 rue Lhomond, \\ F-75231 Paris Cedex 05, France \\ S. Haroche \\ Collège de France, 11 Place Marcelin Berthelot, F-75231 Paris Cedex 05, France \\ E. Jacques, P. Bosland, and B. Visentin \\ DAPNIA, Orme des Merisiers, CEA, F-91191 Gif-sur-Yvette Cedex, France
}

(Received 1 March 2007; accepted 15 March 2007; published online 17 April 2007)

\begin{abstract}
The authors have built a microwave Fabry-Pérot resonator made of diamond-machined copper mirrors coated with superconducting niobium. Its damping time $\left(T_{c}=130 \mathrm{~ms}\right.$ at $51 \mathrm{GHz}$ and $\left.0.8 \mathrm{~K}\right)$ corresponds to a finesse of $4.6 \times 10^{9}$, the highest ever reached for a Fabry-Pérot in any frequency range. This result opens many perspectives for quantum information processing, decoherence and nonlocality studies. (C) 2007 American Institute of Physics. [DOI: 10.1063/1.2724816]
\end{abstract}

Since the Bohr-Einstein photon box thought experiment, storing a photon for a long time has been a dream of physicists. Cavity quantum electrodynamics (CQED) in the microwave domain comes closest to this goal. Photons are trapped in a superconducting cavity and probed by atoms crossing the field one at a time. Experiments with circular Rydberg atoms and Fabry-Pérot resonators have led to fundamental tests of quantum theory and various demonstrations of quantum information procedures. ${ }^{1}$ The open geometry of the cavity is essential to allow a perturbation-free propagation of long-lived atomic coherences through the mode. With this cavity structure, however, the field energy damping time $T_{c}$ is very sensitive to geometrical mirror defects, limiting $T_{c}$ to $\simeq 1 \mathrm{~ms}$ in previous experiments. We report here the realization of a Fabry-Pérot resonator at $\omega / 2 \pi=51 \mathrm{GHz}$, with $T_{c}$ $=130 \mathrm{~ms}$. The cavity quality factor $Q$ is $4.2 \times 10^{10}$ and its finesse $4.6 \times 10^{9}$, the highest ever achieved in any frequency domain for this geometry. This important step opens the way to many CQED experiments. Quantum nondemolition detection of a single photon ${ }^{2}$ and generation of mesoscopic nonlocal quantum superpositions ${ }^{3}$ are now accessible. Long term storage of single photon fields opens bright perspectives for quantum information processing. These high- $Q$ cavities are also promising for the stabilization of microwave oscillators or for the search of exotic particles. ${ }^{4}$

A picture of the cavity $C$ with the top mirror removed is shown in Fig. 1. The mirrors have a diameter $D_{0}=50 \mathrm{~mm}$. The distance between their apexes is $L=27.57 \mathrm{~mm}$. Their surface is toroidal (radii of curvature of 39.4 and $40.6 \mathrm{~mm}$ in two orthogonal planes). The two $\mathrm{TEM}_{900}$ modes near $51.099 \mathrm{GHz}$ with orthogonal linear polarizations are separated by $1.2 \mathrm{MHz}$. This large frequency splitting is essential to ensure that atoms are efficiently coupled to a single mode only. The mirrors are electrically insulated. A static electric field parallel to the cavity axis is applied between them to preserve the circular states and to tune the atomic transition via the Stark effect. ${ }^{1}$ The $1 \mathrm{~cm}$ spacing between mirror edges

\footnotetext{
${ }^{a}$ Present address: J. Gutenberg University, Institute für Physik, Staudingerweg 7, D-55128 Mainz, Germany.

${ }^{b)}$ Electronic mail: jmr@lkb.ens.fr
}

is partly closed by two guard rings improving the static field homogeneity in $C$. The atoms of a thermal beam enter and exit the cavity through two large ports $\left(1 \times 2 \mathrm{~cm}^{2}\right)$ so that they never come close to metallic surfaces, preserving them from patch effect stray fields. This ensures a good transmission of atomic coherences through the cavity. ${ }^{2}$ Four piezoelectric actuators are employed to translate one of the mirrors and to tune the cavity (within $\pm 5 \mathrm{MHz}$ ) with a few hertz accuracy.

We realized two mirror sets, $M_{1}$ and $M_{2}$. The copper substrates are first machined to a gross spherical shape. They are then submitted to two temperature cycles to release stresses. They are first heated in a vacuum chamber to $400{ }^{\circ} \mathrm{C}$ and cooled down to liquid $N_{2}$ temperature. The final diamond machining is then performed (Kugler Company). The local surface roughness is $10 \mathrm{~nm} \mathrm{rms}$ and the surface has a peak-to-valley deviation of $<300 \mathrm{~nm}$ from the ideal shape. To avoid deformation of the mirrors in the final assembly, their thickness is $30 \mathrm{~mm}$. Their back surface and that of the matching holders are polished to $1 \mu \mathrm{m}$.

The mirror surface is covered with a $12-\mu \mathrm{m}$-thick layer of $\mathrm{Nb}$. We use a coating facility at CEA, Saclay, designed for

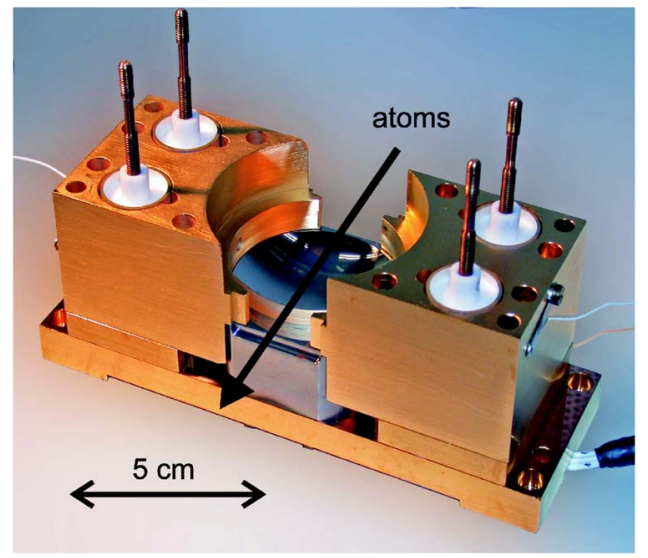

FIG. 1. (Color online) Photograph of the cavity assembly with the top mirror removed. The atomic beam path is visualized by the arrow. The four posts are used to mount the upper mirror. The piezoelectric actuators, centered by the white Teflon cylinders, surround the posts. 
rf cavities used in particle accelerators. ${ }^{5}$ The $\mathrm{Nb}$ layer is deposited by $\mathrm{dc}$ cathode sputtering in a magnetron discharge. ${ }^{6}$ We first clean the substrate with ultrapure filtered alcohol (ultrasonic bath) and dry it with filtered Ar. The sputtering chamber is evacuated to $10^{-8} \mathrm{mbar}$ and then filled with $10^{-1}$ mbar of Ar. We set the mirror potential to $-1000 \mathrm{~V}$ for $20 \mathrm{~s}$, creating an Ar plasma which blows away residual dust particles. The chamber is evacuated again and we start the sputtering process. A $1 \mathrm{~kW}$ magnetron creates a dense $\mathrm{Ar}$ plasma in the vicinity of a cylindrical $\mathrm{Nb}$ cathode (Ar pressure during sputtering: $\left.10^{-3} \mathrm{mbar}\right)$. Initially, the $\mathrm{Nb}$ cathode is far away from the mirror. When its impure surface layer is removed, we move it in front of the mirror. The evaporated atoms are deposited at a rate of $0.1 \mu \mathrm{m} / \mathrm{min}$ on the mirror surface, which heats up to $300-400{ }^{\circ} \mathrm{C}$. Before being mounted in the Rydberg atom setup, the mirrors are finally rinsed in an alcohol ultrasonic bath and dried with Ar.

In order to characterize the cavity modes, microwave is coupled in via weak diffraction loss channels. We thus avoid coupling irises in the mirror centers, which are detrimental to the surface quality. ${ }^{1}$ This coupling is large enough to inject a mesoscopic field in $C$, but not to detect directly the decay of the leaking field. We monitor instead the cavity ring down with an atomic probe. At the beginning of a measurement sequence, we inject a microwave pulse by a waveguide ending in the guard ring around $C$. Most of the microwave power is not coupled into the mode and decays in the apparatus in less than $1 \mu \mathrm{s}$. After a time interval $t$, we send the Rydberg atom probe in $C$. It is produced by a two-step laser excitation of ${ }^{85} \mathrm{Rb}$ atoms involving a diode laser at $420.30 \mathrm{~nm}\left(5 S_{1 / 2} \rightarrow 6 P_{3 / 2}\right)$ and a second diode laser at $1014.67 \mathrm{~nm}\left(6 P_{3 / 2} \rightarrow 52 D_{5 / 2}\right)$. The cavity field induces transitions from $52 D_{5 / 2}$ to other Rydberg levels. The absorption is made broadband by the Stark effect in a $13.4 \mathrm{~V} / \mathrm{cm}$ electric field applied in $C$. Broadband detection is essential for the first cavity tests, since the reproducibility of the mirror mounting results in an uncertainty in the mode frequencies of $\pm 10 \mathrm{MHz}$. The cavity-field-induced atomic transitions are monitored by a state-selective field-ionization detector.

By sweeping the microwave source and recording the atomic absorption, we determine the cavity resonances. For $M_{1}$ and $M_{2}$ we find two modes (lower frequency $M_{i}^{\mathrm{LF}}$ and higher frequency $\left.M_{i}^{\mathrm{HF}}, i=1,2\right)$ close to $51.1 \mathrm{GHz}$, separated by the expected $1.2 \mathrm{MHz}$ splitting. We have mapped the transverse profile of these modes. Using pulsed velocityselected atomic samples, we know the atomic position at any time. Switching the Stark field in $C$ on and off, we set the atoms in resonance during a short time window at a well defined position in $C$. The measured transition probability reveals the intensity profile of the mode along the atomic beam axis. We obtain, as expected, a Gaussian with a $w_{0}$ $\simeq 6 \mathrm{~mm}$ waist.

To measure the quality factors, we record the transition probability $P(t)$ as a function of the delay $t$. Typical data, obtained with $M_{1}^{\mathrm{LF}}$ at $0.8 \mathrm{~K}$ is shown in Fig. 2 for three initial field energies, $E_{0}, e E_{0}$, and $e^{2} E_{0}$ ( $e$ is the base of natural logarithms), corresponding to microwave source attenuations of $8.6,4.3$, and $0 \mathrm{~dB}$, respectively. Due to the exponential decrease of $E$ versus time, $E(t)=E_{0} e^{-t / T_{c}}$, an arbitrary energy $E<E_{0}$ is reached at times $t_{0}, t_{0}+T_{c}$, and $t_{0}$ $+2 T_{c}$ for these three attenuations. Since $P$ depends only on $E$, the corresponding $P(t)$ curves are time shifted by $T_{c}$ with respect to each other. We obtain $T_{c}=112 \pm 4 \mathrm{~ms}$. For $M_{1}^{\mathrm{HF}}, T_{c}$

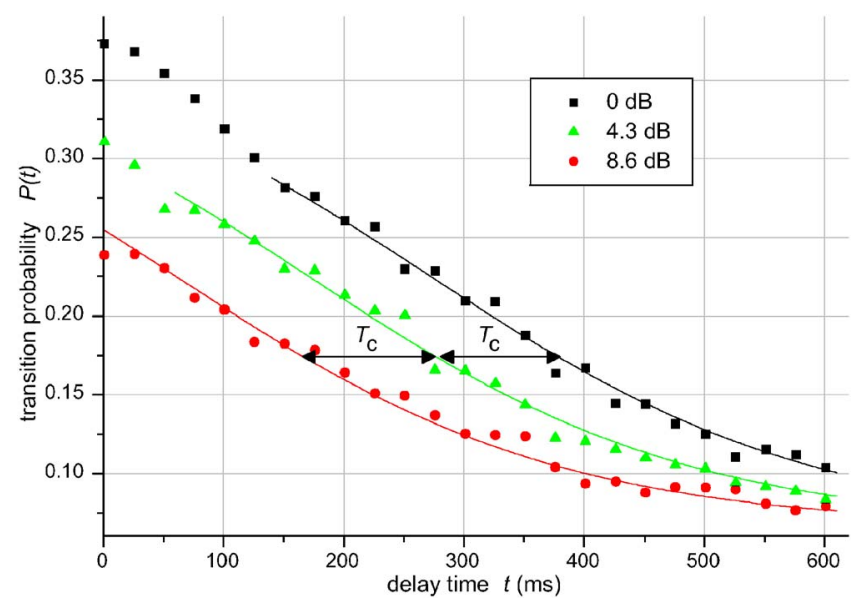

FIG. 2. (Color online) Measurement of $T_{c}\left(M_{1}^{\mathrm{LF}}\right)$ at $T=0.8 \mathrm{~K}$. Transition probability $P(t)$ as a function of the delay $t$ between microwave injection and the atomic probe crossing the cavity. The points (circles, triangles, and squares) correspond to three initial field energies $E_{0}, e E_{0}$, and $e^{2} E_{0}$, respectively. The fits (solid lines) result from a simple absorption model including saturation. They are equidistantly shifted in time with respect to one another by $T_{c}=112 \pm 4 \mathrm{~ms}$. Each point is the average of 1600 atomic detections.

is found to be $87 \pm 10 \mathrm{~ms}$. For $M_{2}^{\mathrm{LF}}$ and $M_{2}^{\mathrm{HF}}$ we get, at $0.8 \mathrm{~K}$, $T_{c}=74 \pm 6 \mathrm{~ms}$ and $T_{c}=130 \pm 4 \mathrm{~ms}$, respectively. These four modes have all an extremely long energy storage time. The longest one corresponds to a light travel distance of $39000 \mathrm{~km}$ folded in the $2.7 \mathrm{~cm}$ space between the mirrors. The corresponding quality factor is $Q=\omega T_{c}=4.2 \times 10^{10}$ and the finesse is $f=Q / 9=4.6 \times 10^{9}$.

We have measured the $M_{2}^{\mathrm{HF}}$ mode spectrum at $0.8 \mathrm{~K}$. The full width at half maximum linewidth is $3 \pm 0.5 \mathrm{~Hz}$, close to the $1.22 \mathrm{~Hz}$ value deduced from $T_{c}$. The difference is due to residual low frequency mechanical vibrations (a $1 \mathrm{~Hz}$ shift corresponds to a $500 \mathrm{fm}$ translation of one mirror). The cavity drift is less than $3 \mathrm{~Hz} / \mathrm{h}$. The stored field coherence is thus well preserved, an important feature for quantum information storage.

We have studied $T_{c}$ as a function of the mirror temperature $T$ for $M_{1}^{\mathrm{LF}}$ (Fig. 3). For $T>1.4 \mathrm{~K}, T_{c}$ increases exponentially versus $1 / T$, while it saturates for $T<1.4 \mathrm{~K}$. The quality factor $Q$ can be expressed as the ratio $G / R_{e}$ of two resistances characterizing the geometry $(G=2800 \Omega$ here $)$ and the

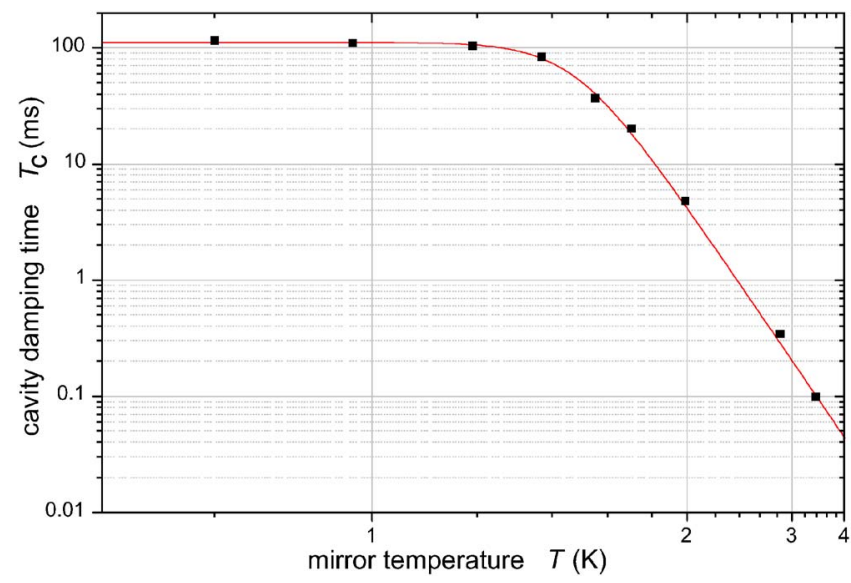

FIG. 3. (Color online) Cavity damping time $T_{c}$ vs mirror temperature $T$ $\left(M_{1}^{\mathrm{LF}}\right)$. The horizontal scale is reciprocal and the vertical one logarithmic. The dots are experimental. The solid line is a fit providing a gap value $\Delta_{0} / k_{B}=20.2 \mathrm{~K}$. Below $1.4 \mathrm{~K}, T_{c}$ saturates at $112 \mathrm{~ms}$. 
losses $\left(R_{e}\right){ }^{7}$ The effective resistance $R_{e}=R_{\mathrm{BCS}}+R_{0}+R_{d}$ has three contributions. The resistance $R_{\mathrm{BCS}}$ is given by BCS theory, the residual resistance $R_{0}$ at $T=0 \mathrm{~K}$ is due to defects and $R_{d}$ measures the diffraction losses. The BCS resistance is $R_{\mathrm{BCS}}=(A / T) \exp \left(-\Delta_{0} / k_{B} T\right)$, where $\Delta_{0}$ is the superconducting gap and $A$ is intrinsic to $\mathrm{Nb}$. For $T>1.6 \mathrm{~K}, R_{\mathrm{BCS}}$ dominates $R_{e}$, while it is negligible for $T<1.4 \mathrm{~K}$. The line in Fig. 3 is a fit with $T_{c}=G / \omega R_{e}$, from which we infer $\Delta_{0} / k_{B}$ $=20.2 \pm 0.3 \mathrm{~K}$. This value, confirmed by measurements on $M_{1}^{\mathrm{HF}}$ and $M_{2}$, differs slightly from the value $17.056 \mathrm{~K}$ found at $22 \mathrm{GHz}$ with bulk $\mathrm{Nb}$ in Ref. 9 .

The saturation of $T_{c}$ below $1.4 \mathrm{~K}$ yields $R_{0}+R_{d}=75 n \Omega$ for $M_{1}^{\mathrm{LF}}$ and $68 n \Omega$ for $M_{2}^{\mathrm{HF}}$. The order of magnitude of the diffraction losses $R_{d}$ can be estimated. The mirror diameter $D_{0}$ limits $Q$ to $Q_{\text {diff }}=(\omega L / c) \exp \left(D_{0}^{2} / 2 w^{2}\right)=2.7 \times 10^{11}(w$ $=1.23 w_{0}$ is the mode waist at the mirror surface). The surface roughness is characterized by the rms deviation $h_{\mathrm{rms}}$ with respect to the ideal shape. The corresponding quality factor, calculated by evaluating the "total integrated scattering,", is $Q_{\text {surf }}=c L / 4 \omega h_{\mathrm{rms}}^{2}$. From the measured $h_{\mathrm{rms}} \simeq 10 \mathrm{~nm}$, we obtain $Q_{\text {surf }}=6.4 \times 10^{10}$. Combining these losses yields $Q^{\prime}$ $=\left(Q_{\text {diff }}^{-1}+Q_{\text {surf }}^{-1}\right)^{-1}=5.2 \times 10^{10}$. This is close to the best measured value $\left(Q=4.2 \times 10^{10}\right)$, indicating that diffraction losses are the dominant contribution at low $T$.

It is also instructive to compare the $Q$ factor of our open resonators with that of closed cylindrical cavities at $22 \mathrm{GHz}$ used in Rydberg atom micromaser studies. ${ }^{9,10}$ In these experiments, $Q=4.0 \times 10^{10}$ at $0.3 \mathrm{~K}$ was measured. ${ }^{11}$ The residual resistance was $R_{0}=G / Q=28 n \Omega(G=1089 \Omega$-there are no diffraction losses in this geometry), a value comparable to ours. The difference could be due to the diffraction losses in our open geometry, to the frequency dependence of $R_{0}$, or to the variations in the $\mathrm{Nb}$ purity. It is remarkable that our open cavity reaches the same $Q$ as the best closed one in the same frequency domain. At much lower frequencies, around $1 \mathrm{GHz}, Q$ factors up to $10^{12}$ have been obtained. ${ }^{12}$ This frequency domain is, however, much less convenient for cavity QED experiments.
We have reported the realization of a ultrahigh- $Q$ photon box. This cavity, with its open geometry, is ideally suited for the propagation of atomic coherence through the field mode, atomic interferometry, decoherence studies, and quantum information processing experiments. Experiments with two such cavities are of particular interest. A single atom could be used to entangle two mesoscopic fields separated by a macroscopic distance, resulting in the preparation of a nonlocal quantum state. ${ }^{3}$

The authors acknowledge support by the DGA, by the Japan Science and Technology Agency (JST), by the EU under the IP projects "QGATES" and "SCALA," and by a Marie-Curie fellowship of the European Community to one of the authors (S.K.). The authors thank J. Olejnik for the design of the set-up.

${ }^{1}$ J. M. Raimond, M. Brune, and S. Haroche, Rev. Mod. Phys. 73, 565 (2001).

${ }^{2}$ S. Gleyzes, S. Kuhr, C. Guerlin, J. Bernu, S. Deléglise, U. Busk Hoff, M. Brune, J. M. Raimond, and S. Haroche, Nature (London) 446, 297 (2007); e-print quant-ph/0612031.

${ }^{3}$ P. Milman, A. Auffeves, F. Yamaguchi, M. Brune, J. M. Raimond, and S. Haroche, Eur. Phys. J. D 32, 233 (2005).

${ }^{4}$ R. Bradley, J. Clarke, D. Kinion, L. J. Rosenberg, K. Van Bibber, S. Matsuki, M. Mück, and P. Sikivie, Rev. Mod. Phys. 75, 777 (2003).

${ }^{5}$ P. Bosland, A. Aspart, E. Jacques, and M. Ribeaudeau, IEEE Trans. Appl. Supercond. 9, 896 (1999)

${ }^{6}$ C. Benvenuti, S. Calatroni, I. E. Campisi, P. Darriulat, M. A. Peck, R. Russo, and A. M. Valente, IEEE Trans. Appl. Supercond. 9, 900 (1999).

${ }^{7}$ J. Halbritter, J. Appl. Phys. 42, 82 (1971).

${ }^{8}$ W. Winkler, R. Schilling, K. Danzmann, J. Mizuno, A. Rüdiger, and K. A. Strain, Appl. Opt. 33, 7547 (1994).

${ }^{9}$ D. Meschede, H. Walther, and G. Müller, Phys. Rev. Lett. 54, 551 (1985).

${ }^{10}$ G. Rempe, F. Schmidt-Kaler, and H. Walther, Phys. Rev. Lett. 64, 2783 (1990).

${ }^{11}$ S. Brattke, B. T. H. Varcoe, and H. Walther, Phys. Rev. Lett. 86, 3534 (2001).

${ }^{12}$ V. Arbert-Engels, C. Benvenuti, S. Calatroni, P. Darriulat, M. A. Peck, A. M. Valente, and C. A. Van't Hof, Nucl. Instrum. Methods Phys. Res. A 463, 1 (2001). 